\title{
Thermodynamic and transport properties of underdoped cuprates from ARPES data
}

\author{
T. Yoshida ${ }^{\text {a,* }}$ X. J. Zhou ${ }^{\text {a }}$, H. Yagi ${ }^{\text {b }}$, D. H. Lu ${ }^{\text {a }}$, K. Tanaka ${ }^{\text {, }}$, \\ A. Fujimori ${ }^{\text {b }}$ Z. Hussain ${ }^{\text {c }}$ Z.-X. Shen ${ }^{\text {a }}$, T. Kakeshita ${ }^{\text {d,e }}$, \\ H. Eisaki ${ }^{\mathrm{f}}$, S. Uchida ${ }^{\mathrm{e}}$, Kouji Segawa ${ }^{\mathrm{g}}$, A.N. Lavrov ${ }^{\mathrm{g}}$ and \\ Yoichi Ando ${ }^{g}$ \\ ${ }^{a}$ Department of Applied Physics and Stanford Synchrotron Radiation Laboratory, \\ Stanford University, Stanford, CA94305, USA \\ ${ }^{\mathrm{b}}$ Department of Physics and Department of Complexity Science and Engineering, \\ University of Tokyo, Bunkyo-ku, Tokyo 113-0033, Japan \\ ${ }^{c}$ Advanced Light Source, Lawrence Berkeley National Lab, Berkeley, CA 94720, \\ $U S A$ \\ d Superconductivity Research Laboratory, ISTEC, Shinonome 1-10-13, Koto-ku, \\ Tokyo 135-0062, Japan \\ e Department of Physics, The University of Tokyo Tokyo 113-8656, Japan \\ ${ }^{\mathrm{f}}$ National Institute of Advanced Industrial Science and Technology, Tsukuba \\ 305-8568, Japan \\ ${ }^{g}$ Central Research Institute of Electric Power Industry, Komae, Tokyo 201-8511, \\ Japan
}

\begin{abstract}
The relationship between photoemission spectra of high- $T_{\mathrm{C}}$ cuprates and their ther-
\end{abstract}

Preprint submitted to Elsevier Science

6 November 2018 
modynamic and transport properties are discussed. The doping dependence of the expected quasi-particle density at the Fermi level $\left(E_{\mathrm{F}}\right)$ are compared with the electronic specific heat coefficient $\gamma$ and that of the spectral weight at $E_{\mathrm{F}}$ with the in-plane and out-of-plane superfluid density. We have estimated the electrical resistivity of underdoped cuprates from the momentum distribution curve (MDC) at $E_{\mathrm{F}}$ in the nodal direction. The temperature dependence of the MDC width is also consistent with that of the electrical resistivity.

Key words: Angle-resolved photoemission, high- $T_{\mathrm{C}}$ cuprates, specific heats, electrical resisitivity

\section{Introduction}

Thermodynamics and the transport properties of high- $T_{\mathrm{C}}$ cuprates are highly affected by the strong electron correlation near the filling-control Mott transition. One of the most unconventional thermodynamic properties is that the electronic specific heat coefficient $\gamma$ shows pseudogap phenomena in the underdoped region [1]. The electronic resistivity show unconventional features such as $T$-liner temperature dependence in the optimally doped region and metallic behavior well exceeding the Ioffe-Regel limit in the lightly doped region [2]. In order to elucidate the origin of these unconventional phenomena, angle-resolved photoemission spectroscopy (ARPES) is an extremely powerful technique because one can directly observe the spectral function $A(\mathbf{k}, \omega)$,

* Teppei Yoshida +1-650-725-5457(FAX)

McCullough Bldg. Rm. 220, 476 Lomita Mall, Stanford University, Stanford, CA 94305, USA

Email address: teppei@stanford.edu (T. Yoshida). 
i.e., the electronic structure, near the Fermi level $\left(E_{\mathrm{F}}\right)$. Therefore, information obtained from ARPES can be directly compared with thermodynamic properties such as the electronic specific heat. Also, the ARPES spectra near $E_{\mathrm{F}}$ give significant information about the transport properties. In this article, we shall attempt to understand the unconventional thermodynamics and transport properties based on ARPES spectra mostly just at $E_{\mathrm{F}}[3]$.

\section{Thermodynamic properties}

The electronic specific heat coefficient $\gamma$ of a Fermi liquid is given by $\gamma=$ $\pi^{2} k_{B}^{2} / 3 N(0)^{*}$, where $N(0)^{*}$ is the quasi-particle (QP) density at $E_{\mathrm{F}}$. In a single-band system like single-layer cuprates, each momentum contributes one QP, if the system is a Fermi liquid. Therefore, if one successfully fits the dispersion of the QP band to some energy band model such as the tightbinding (TB) model, the density of states (DOS) of that band yields $N(0)^{*}$.

In Fig. 4(a), the DOS determined by a TB fit to the ARPES results of $\mathrm{La}_{2-x} \mathrm{Sr}_{x} \mathrm{CuO}_{4}$ (LSCO) is compared with the specific heat coefficient $\gamma$ of LSCO samples where the $T_{\mathrm{C}}$ is suppressed by Zn-doping [1]. The TB parameters have been determined to reproduce the experimentally observed Fermi surface and the slope of the band dispersions in the nodal $(0,0)-(\pi, \pi)$ direction. Here, fine structures of "kink" in the energy dispersions [4] are neglected in the TB fit. The TB band DOS has a maximum around $x=0.15$ because the flat band near $(\pi, 0)$ becomes closest to the Fermi level. If the system is a Fermi liquid, the TB band DOS and $\gamma$ should be the same. However, the $\gamma$ decreases much faster than TB band DOS with decreasing $x$. This difference

indicates that the QP density at $E_{\mathrm{F}}$ that would be expected for a normal 
Fermi liquid is indeed depleted in the underdoped materials and demonstrates how the opening of the pseudo-gap occurs around $(\pi, 0)$.

The doping dependence of the DOS of angle integrated photoemission (AIPES) spectra at $E_{\mathrm{F}}$ are also shown in Fig. 4(a). AIPES spectra have been obtained by integrating the ARPES spectra with respect to $\mathbf{k}$ over the Brillouin zone. As a whole, the AIPES DOS decreases with decreasing $x$ for $x<0.22$ due to the pseudo-gap opening. However, a closer inspection reveals that the AIPES DOS decreases faster than $\gamma$ with decreasing $x$. Note that the DOS at $E_{\mathrm{F}}$ measured by AIPES is given by $z N(0)^{*}$, where $z$ is the renormalization factor. The faster decrease of the AIPES intensity with decreasing $x$ is due to the effects of $z$, which also decreases with decreasing $x$ [5]. From the above comparison, one can say that the AIPES DOS reflects the effects of both the pseudogap opening and the spectral weight renormalization $z$.

Relationship between the ARPES spectral weight and the superfluid density would also give important key about the superconductivity [6]. The doping dependence of the ARPES spectral weight at $E_{\mathrm{F}}$ is compared with that of the penetration depth $1 / \lambda^{2} \sim \rho_{s} / m^{*}[7]$ in Fig. 4(b). The spectral weight at angle $\alpha$ (see inset) is defined by the integrated spectral weight of the spectral function $A(\mathbf{k}, \omega)$ at $E_{\mathrm{F}}$ over the momentum.

The decrease of both the in-plane and out-of plane superfluid densities for $x<0.20$ is due to the pseudogap opening near $(\pi, 0)$. Particularly, the doping dependence of the out-of-plane $\left(\rho_{s} / m^{*}\right)_{c} \sim 1 / \lambda_{c}^{2}$ well corresponds to that of the spectral weight around $\sim(\pi, 0) \quad\left(\alpha=0^{\circ}\right)$. This is because the out-ofplane $\left(\rho_{s} / m^{*}\right)_{c} \sim 1 / \lambda_{c}^{2}$ has large matrix element around $(\pi, 0)$. On the other hand, the nodal direction is expected to be the main contribution to in-plane 
$\left(\rho_{s} / m^{*}\right)_{a b} \sim 1 / \lambda_{a b}^{2}$ because of the lighter effective mass. However, the decrease with decreasing $x$ is somewhat different from the doping dependence of the nodal spectral weight $\left(\alpha=45^{\circ}\right)$. This implies that not only the nodal QP's but also QP's over an extended $k$-region of the "Fermi arc" condense into the superfluid.

\section{Transport properties}

The transport properties of high- $T_{\mathrm{C}}$ cuprates show unconventional behavior reflecting the strongly electron correlation. Because of the pseudo-gap opening around $(\pi, 0)$, the electronic states around the node mainly contribute to the inplane transport properties in the underdoped region. This portion of the Fermi surface survives in the upderdoped compounds as a "Fermi arc" [5]. We shall estimate the in-plane electrical resistivity from the observed "Fermi arc" using the conventional Drude formula $\rho=m^{*} / n e^{2} \tau$. Here, the renormalized mass $m^{*}=\hbar k_{\mathrm{F}} / v_{\mathrm{F}}$ can be determined from the quantities measured by ARPES, namely, the Fermi velocity $v_{\mathrm{F}}$ and the Fermi momentum $k_{\mathrm{F}}$ of the hole-like Fermi surface centered at $(\pi, \pi)$. From the MDC width $\Delta k$ at $E_{\mathrm{F}}$, the mean free path is obtained as $l=1 / \Delta k$, yielding the mean free scattering time of $\tau=l / v_{\mathrm{F}}$. Thus, one can evaluate $\rho=m^{*} / n e^{2} \tau=\hbar k_{\mathrm{F}} \Delta k / n e^{2}$ from ARPES spectra.

Figure 4(a) shows an ARPES intensity plot in the $E$ - $k$ space for an LSCO $(x$ $=0.03)$ sample in the $(0,0)-(\pi, \pi)$ direction. The energy dispersion determined by the peaks in the MDC shows a "kink" feature around $70 \mathrm{meV}$ below $E_{\mathrm{F}}$, which is presumably due to electron-phonon coupling [4]. $v_{\mathrm{F}} \propto 1 / m^{*}$ in the node direction is almost doping independent $v_{\mathrm{F}} \sim 1.8 \mathrm{eV} \AA$ for LSCO [8]. 
Now, we discuss how to estimate the carrier number $n$. Although the Fermiliquid picture yields the Fermi surface volume $\sim 1-x$, Hall coefficient results show unconventional behavior $n \sim x$ in the underdoped region [9]. The observed "Fermi arc" picture gives two possibilities to explain the evolution of the carrier number like $n \sim x$ [5]. One possibility is that the length of the "Fermi arc" increases with $x$ and yields $n \sim x$. However, the "arc" length estimated from the ARPES intensity does not change enough to explain the $n \sim x$ behavior. Another possibility is the evolution of the nodal spectral weight with $x$. Indeed, the spectral weight as a function of $x$ is remarkably similar to the $x$-dependence of the Hall coefficient [5].

Figure $4 \mathrm{~b}$ shows the doping dependence of the estimated in-plane electrical resistivity $\rho_{\mathrm{ARPES}}$ based on the Drude formula by using the parameters $k_{\mathrm{F}}$, $\Delta k$ and $n$ as described above. ARPES intensity $n_{\mathrm{PES}} \sim n_{\text {Hall }}, x$ ("small Fermi surface volume") and $1-x$ ("large Fermi surface volume") are considered as the carrier number $n$. $\rho_{\text {ARPES }}$ evaluated using $n=x$ and $n_{\text {PES }}$ are in good agreement with the transport results in the underdoped region. On the other hand, $\rho_{\text {ARPES }}$ evaluated using $n=1-x$ is by more than one order of magnitude smaller than the transport data in the underdoped region. This suggests that the pseudogap and the small renormalized spectral weight is the origin of the small carrier number and the high electrical resistivity for the underdoped samples. On the other hand, the discrepancy between $\rho_{\text {ARPES }}\left(n=x, n_{\mathrm{PES}}\right)$ and the transport data becomes pronounced in the optimally doped region. This discrepancy may indicate a cross-over from the Fermi arc to the large Fermi surface and/or may be due to the contribution from the $(\pi, 0)$ region, which is not precisely taken into account in the present simplified analysis. We leave such quantitative analysis for future investigation. As a whole, the 
estimated $\rho_{\text {ARPES }}$ for $n_{\mathrm{PES}}$ explains the doping dependence of the transport data of the underdoped LSCO qualitatively well.

We also show the case of $\mathrm{YBa}_{2} \mathrm{Cu}_{3} \mathrm{O}_{y}(\mathrm{YBCO})$ in Fig. 4(a)(b). Relationship between the oxygen content $y$ and the hole concentration $\delta$ has been estimated from the electrical resistivity and the thermopower [10]. The MDC peak width of YBCO is about twice as large as that of LSCO and the MCD line shape of YBCO could not be well fitted to a single Lorentzian. We consider that this is due to the bilayer splitting in the nodal direction as predicted by band-structure calculations [11]. We have therefore fitted the MDC to two Lorentzians to estimate $\Delta k$, assuming that the chain band does not exist at $E_{\mathrm{F}}$ for such low doping. Fig.4(b) shows the estimated value of $\rho$ in the same way as Fig.4(b). While the transport data $[12,13]$ is closer to $\rho_{\text {ARPES }}(n=\delta)$ for $\delta=0.04$, it approaches $\rho_{\mathrm{ARPES}}(n=1-\delta)$ with increasing $\delta$, again implying the crossover from the Fermi arc to the large Fermi surface.

Because the Fermi velocity $v_{\mathrm{F}}$ does not seem to change with temperature, the temperature dependence of the inverse life time $1 / \tau$ is determined by that of $\Delta k=1 / l=1 / v_{\mathrm{F}} \tau$. Figure $4(\mathrm{a})$ shows the temperature dependence of MDC at $E_{\mathrm{F}}$ in the nodal direction for the $x=0.07$ sample. The MDC width $\Delta k$ is systematically broadened with increasing temperature, indicating a decrease of the mean-free path. As shown in Fig.4(b), the temperature dependence of $\Delta k$ well scales with that of the electric resistivity, indicating that the temperature dependence of $\Delta k$ determines that of the resistivity. In optimally doped Bi2212, the linear temperature dependence of the MDC width which scales with resistivity was observed and discussed in the context of marginal Fermi-liquid picture [14]. The present result also shows a $T$-linear behavior in $\Delta k$ over a wide temperature range. However, considering that electron-phonon 
interaction causes the "kink" behavior [4] and contribute to the low energy properties, the MDC width would be affected by phonons.

\section{Conclusion}

In summary, we have discussed the relationship between the ARPES results for high- $T_{\mathrm{C}}$ cuprates and their thermodynamics and transport properties. From the comparison of the ARPES and $\gamma$ and $\rho_{s}$, the pseudogap feature and the spectral weight renormalization are discussed. We have estimated the electric resistivity from the observed spectra and have demonstrated that the obtained results explain the transport properties. The temperature dependence of the MDC width $\Delta k$ in the nodal direction well scales with that of the electric resistivity.

\section{Acknowledgements}

We are grateful to H. Fukuyama for enlightening discussions. This work was supported by a Grant-in-Aid for Scientific Research "Novel Quantum Phenomena in Transition Metal Oxides" from the Ministry of Education, Science, Culture, Sports and Technology of Japan and the New Energy and Industrial Technology Development Organization (NEDO). Experimental data were recorded at ALS and SSRL which are operated by the Department of Energy's Office of Basic Energy Science, Division of Material Sciences. SSRL is also operated by Division of Chemical Sciences. 


\section{References}

[1] N. Momono et al., Physica C 233 (1994) 395.

[2] Y. Ando et al., Phys. Rev. Lett. 87 (2001) 017001.

[3] The ARPES data presented here were measured at BL10.0.1.1 of the Advanced Light Source (ALS) and BL 5-4 of the Stanford Synchrotron Radiation Laboratory (SSRL). The detail of the experimental conditions are explained in T. Yoshida et al., Phys. Rev. B 63 (2001) 220501.

[4] A. Lanzara et al., Nature 412 (2001) 510.

[5] T. Yoshida et al., Phys. Rev. Lett. 91 (2003) 027001.

[6] D. L. Feng et al., Science 289 (2000) 277.

[7] C. Panagopoulos et al., Phys. Rev. B 67 (2003) 220502.

[8] X.-J. Zhou et al., Nature 423 (2003) 398.

[9] H. Takagi et al., Phys. Rev. Lett. 69 (1992) 2975.

[10] S. Uchida and N. P. Ong, unpublished.

[11] O. K. Andersen et al., Phys. Rev. B 49 (1994) 4145.

[12] Y. Ando, A. N. Lavrov, and K. Segawa, Phys. Rev. Lett. 83 (1999) 2813.

[13] Y. Ando, K. Segawa, S. Komiya, and A. N. Lavrov, Phys. Rev. Lett. 88 (2002) 137005.

[14] T. Valla et al., Science 285 (1999) 2110. 
Fig. 1 (a) Comparison the electronic specific heat coefficient $\gamma$ [1] and the TB band DOS at $E_{F}$. The doping dependence of AIPES DOS at $E_{\mathrm{F}}$ is also shown. (b) Doping dependence of the spectral weight around nodal and $(\pi, 0)$ regions. The in-plane and out-of-plane superfluid densities deduced from the magnetic penetration depth $\left(\lambda_{a b}, \lambda_{c}\right)[7]$ are compared with them.

Fig. 2 (a) Spectral intensity in the $E-k$ space along the nodal cut and the MDC at the Fermi level. (b) Comparison of the electrical resistivity at $20 \mathrm{~K}$ [2] and the resistivity estimated from ARPES. The carrier number $n$ is assumed to be $n=x, n=1-x$ and $n=n_{\mathrm{PES}}$.

Fig. 3 (a) Spectral intensity of YBCO in the $E-k$ space along the nodal cut and the MDC at the Fermi level. (b) Comparison of the electrical resistivity at $10 \mathrm{~K}[12,13]$ and the resistivity estimated from ARPES. The carrier number $n$ is assumed to be $n=\delta$ and $n=1-\delta$.

Fig. 4 (a) Temperature dependence of the MDC at $E_{\mathrm{F}}$ in the nodal direction for $x=0.07$. (b) Comparison of the temperature dependence of the MDC momentum width $\Delta k$ with that of the electrical resistivity. 


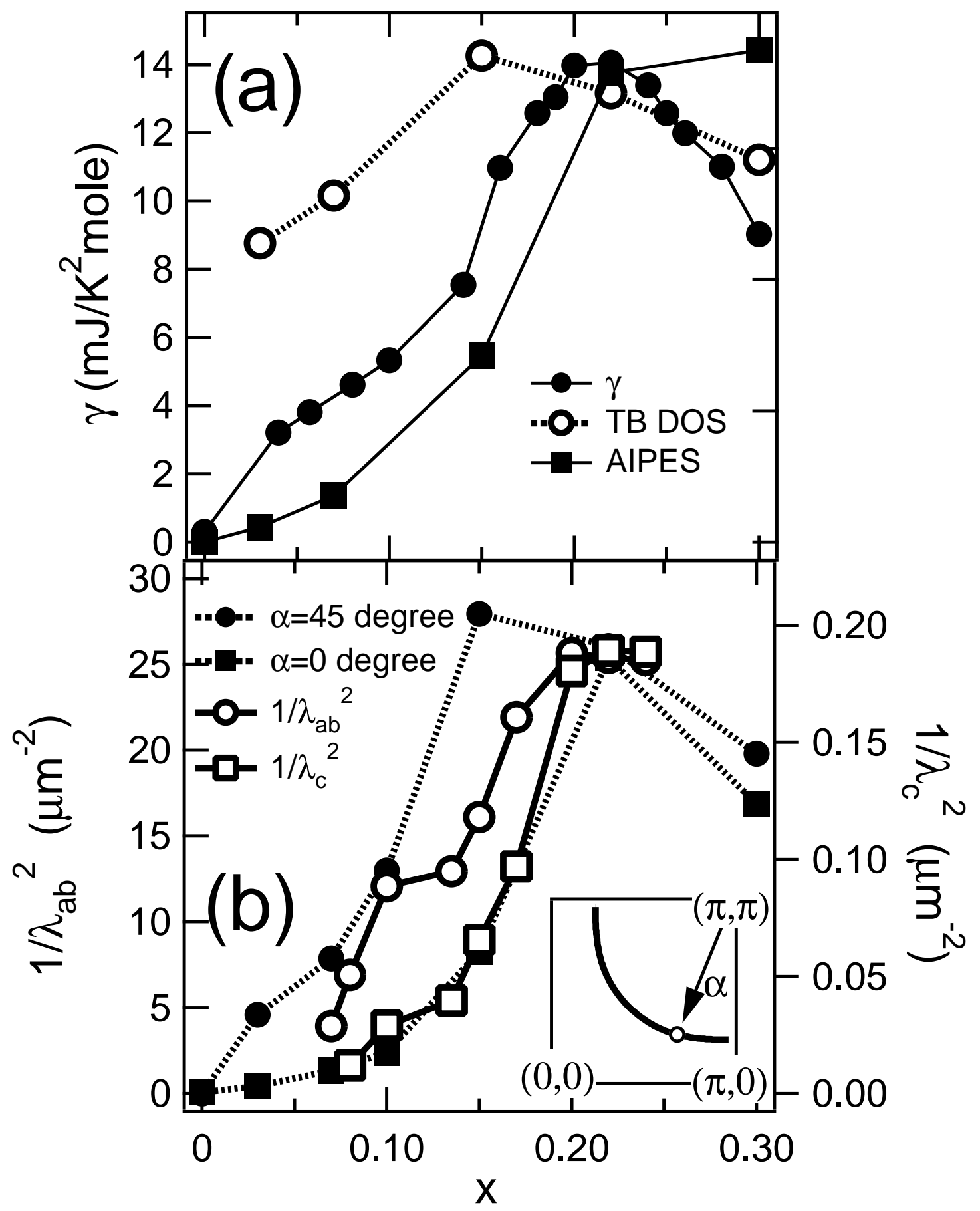

Fig. 1 

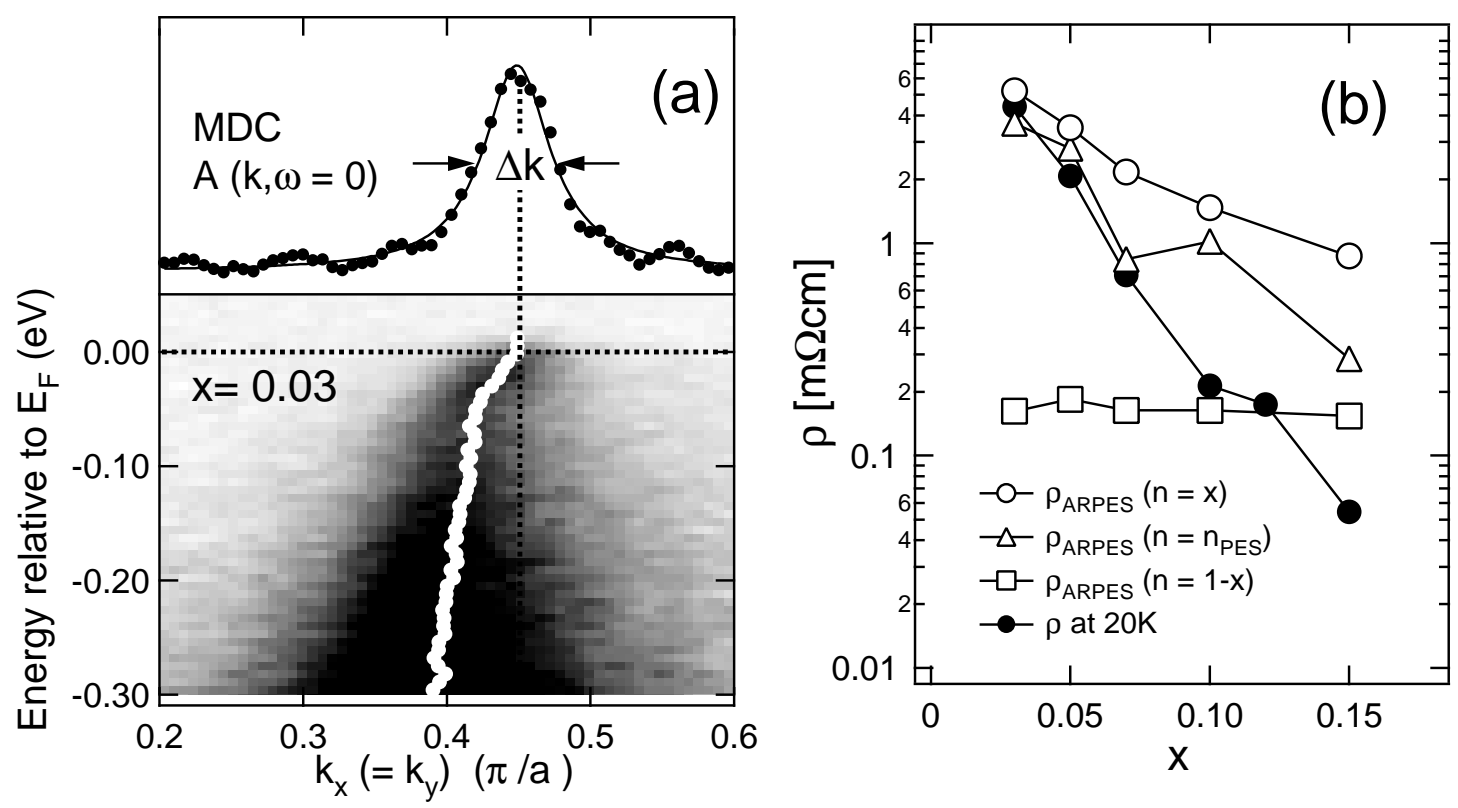

Fig. 2 

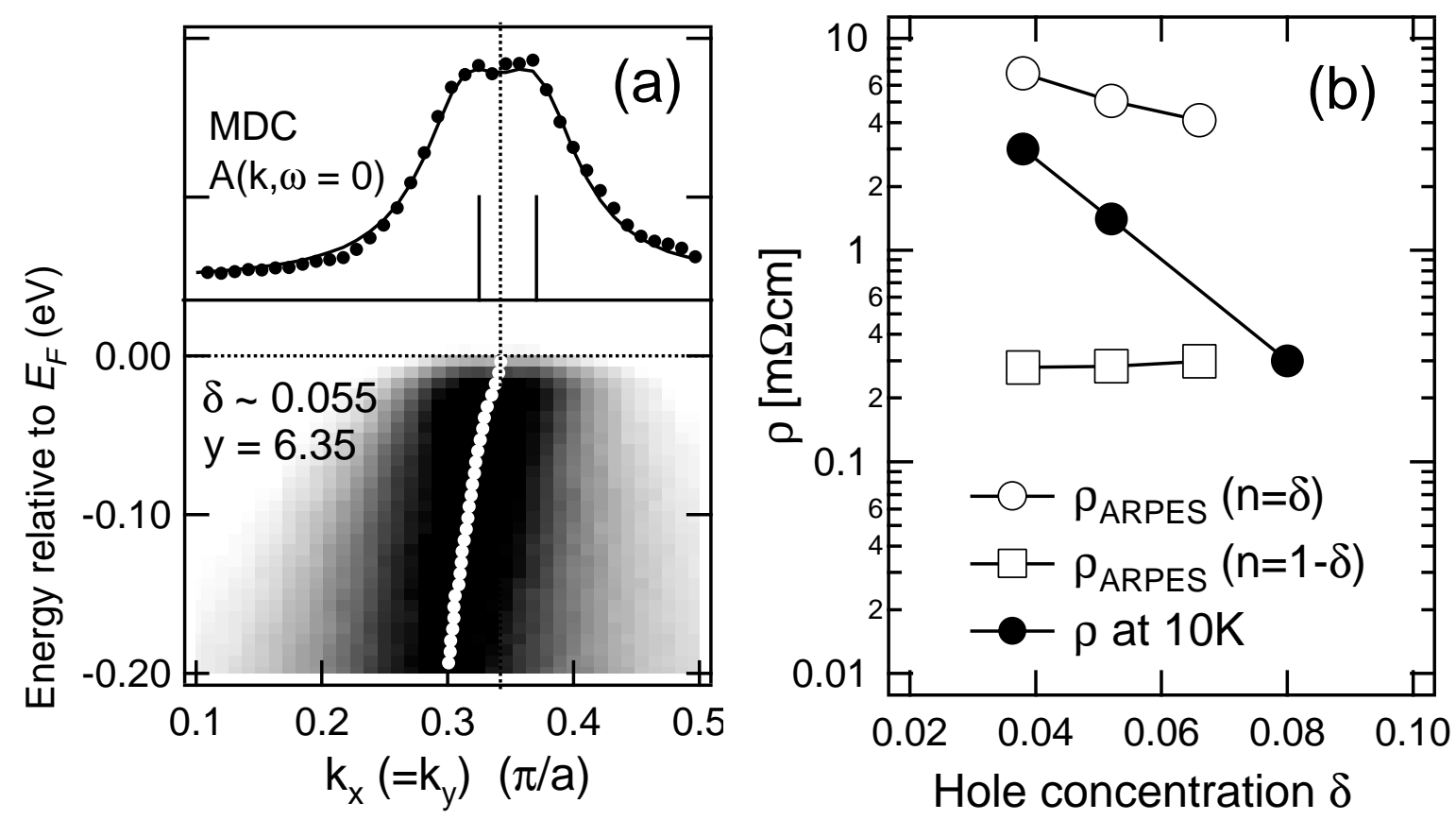

Fig. 3 

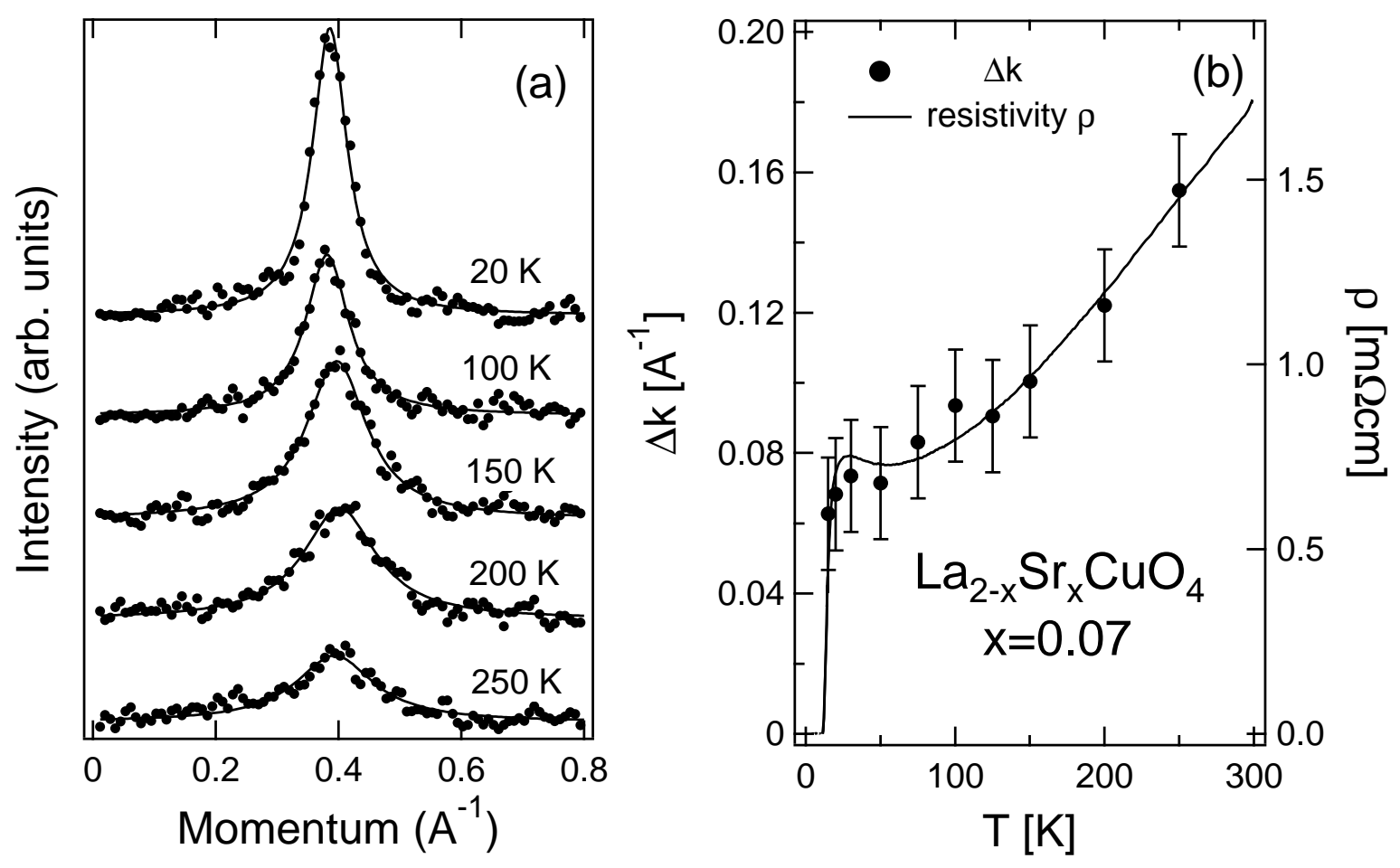

Fig. 4 